\title{
Smad2/4 Signaling Pathway Is Critical for Epidermal Langerhans Cell Repopulation Under Inflammatory Condition but Not Required for Their Homeostasis at Steady State
}

OPEN ACCESS

Edited by:

Thomas Marichal,

University of Liège, Belgium

Reviewed by:

Florian Sparber,

University of Zurich, Switzerland Clare L. Bennett,

University College London,

United Kingdom

Botond Z. Igyártó,

Thomas Jefferson University,

United States

*Correspondence:

Zhirong Yao

yaozhirong@xinhuamed.com.cn Qing-Sheng Mi

QMl1@hfhs.org

tThese authors have contributed equally to this work

Specialty section:

This article was submitted to Antigen Presenting Cell Biology,

a section of the journal

Frontiers in Immunology

Received: 24 November 2019

Accepted: 20 April 2020

Published: 07 May 2020

Citation:

Huang L, Li G-H, YU Q, XU Y, Cvetkovski S, Wang $X$, Parajuli N, Udo-Inyang I, Kaplan D, Zhou L, Yao Z and Mi Q-S (2020) Smad2/4 Signaling

Pathway Is Critical for Epidermal Langerhans Cell Repopulation Under Inflammatory Condition but Not

Required for Their Homeostasis at Steady State. Front. Immunol. 11:912.

doi: 10.3389/fimmu.2020.00912

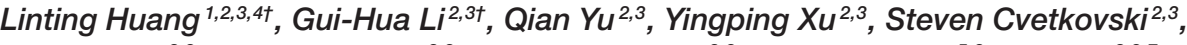
Xuan Wang ${ }^{2,3}$, Nirmal Parajuli ${ }^{2,3}$, Imo Udo-Inyang ${ }^{2,3}$, Daniel Kaplan ${ }^{5,6}$, Li Zhou $^{2,3,7}$, Zhirong Yao ${ }^{1,4 *}$ and Qing-Sheng $\mathrm{Mi}^{2,3,7 *}$
\end{abstract}

${ }^{1}$ Department of Dermatology, Xinhua Hospital, Shanghai Jiao Tong University School of Medicine, Shanghai, China, ${ }^{2}$ Center for Cutaneous Biology and Immunology, Department of Dermatology, Henry Ford Health System, Detroit, MI, United States, ${ }^{3}$ Immunology Research Program, Henry Ford Cancer Institute, Henry Ford Health System, Detroit, MI, United States, ${ }^{4}$ Institute of Dermatology, Shanghai Jiao Tong University School of Medicine, Shanghai, China, ${ }^{5}$ Department of Dermatology, University of Pittsburgh, Pittsburgh, PA, United States, ${ }^{6}$ Department of Immunology, University of Pittsburgh, Pittsburgh, PA, United States, ${ }^{7}$ Department of Internal Medicine, Henry Ford Health System, Detroit, MI, United States

Epidermal Langerhans cells (LCs) are skin-resident dendritic cells that are essential for the induction of skin immunity and tolerance. Transforming growth factor- $\beta 1$ (TGF $\beta 1$ ) is a crucial factor for LC maintenance and function. However, the underlying TGF $\beta 1$ signaling pathways remain unclear. Our previous research has shown that the TGF $\beta 1 / \mathrm{Smad} 3$ signaling pathway does not impact LC homeostasis and maturation. In this study, we generated mice with conditional deletions of either individual Smad2, Smad4, or both Smad2 and Smad4 in the LC lineage or myeloid lineage, to further explore the impact of TGF $\beta 1 / \mathrm{Smad}$ signaling pathways on LCs. We found that interruption of Smad2 or Smad4 individually or simultaneously in the LC lineage did not significantly impact the maintenance, maturation, antigen uptake, and migration of LCs in vivo or in vitro during steady state. However, the interruption of both Smad2 and Smad4 pathways in the myeloid lineage led to a dramatic inhibition of bone marrow-derived LCs in the inflammatory state. Overall, our data suggest that canonical TGF $\beta 1 / S m a d 2 / 4$ signaling pathways are dispensable for epidermal LC homeostasis and maturation at steady state, but are critical for the long-term LC repopulation directly originating from the bone marrow in the inflammatory state.

Keywords: Langerhans cells, Smad2, Smad3, Smad4, transforming growth factor- $\beta 1$

\section{INTRODUCTION}

Epidermal Langerhans cells (LCs) functionally serve as a unique subset of skin-resident dendritic cells (DCs), although they are also classified as a specialized macrophage subset in skin based on their developmental relationship like tissue resident macrophage derived from embryonic precursors (1). LCs represent the first antigen-presenting cells in the skin that encounter foreign 
antigens and take part in a variety of immunological processes. "Classical" epidermal LCs, characterized by the expression of a C-type lectin receptor known as Langerin (CD207), are defined as $\mathrm{CD}^{+} 5^{+} \mathrm{MHCII}^{+}$Langerin $^{+}$cells (2-5). LCs phagocytize epidermal detritus with their dendritic protrusions and eventually present these antigens on the cell surface with an MHCII molecule (6). Following cell activation by various stimuli, LCs have increased expression of costimulatory markers CD80 and CD86, decreased expression of epithelial cell adhesion molecule (EpCAM) and E-cadherin, and acquire a migratory phenotype. These changes result in LC migration to the regional draining lymph nodes (LNs), where the LCs interact with naive $\mathrm{T}$ cells to induce immunity or tolerance $(3,7)$. At steady state, LCs self-renew, while under the inflammatory state, such as ultraviolet (UV) light exposure-induced inflammation, there are two waves of LC repopulation in the skin (8). The first wave involves fast "short-term" LCs that are derived from peripheral blood Gr- $1^{\text {hi }}$ monocytes and express low levels of Langerin, while the second wave generates "long-term" LCs that are derived from bone marrow (BM) and express high levels of Langerin (8). Despite recent research advances, the specific underlying mechanisms that precisely regulate $\mathrm{LC}$ development and function are still not fully understood.

Transforming growth factor- $\beta$ (TGF $\beta$ ) is a key cytokine for the differentiation and maintenance of LCs. The regulatory roles of TGF $\beta 1$ include promoting LC development, inhibiting LC maturation and migration to draining LNs (9), and regulating LC repopulation during inflammation (8). LCs are absent in mice that lack TGF $\beta 1$ due to failure of LC differentiation, survival, or both (9-11). Although TGF $\beta 1$ is expressed by both keratinocytes and LCs, an autocrine source of TGF $\beta 1$ is required for LC development (9). However, the underlying mechanisms of TGF $\beta 1$ signaling in LCs remain elusive. In the classical TGF $\beta 1$ signaling pathway, TGF $\beta 1$ signals through TGF $\beta$ receptor 2 (TGF $\beta$ R2) and TGF $\beta$ receptor 1 (TGF $\beta R 1$ ). Subsequently, these receptors activate two structurally-similar proteins, Smad2 and Smad3, which leads to the formation of a heterotrimeric complex with a common Smad4 that translocates to the nucleus to regulate TGF $\beta 1$ target genes $(12,13)$. Some Smad-independent pathways, such as Erk, JNK, and p38 MAPK kinase, also mediate TGF 1 signaling $(13,14)$. Given the ubiquitous influence of TGF $\beta R /$ Smad signaling on immune regulation $(13,15)$, we sought to investigate how Smad2/3/4 proteins in the TGF $\beta 1$ pathway regulate $\mathrm{LC}$ development.

Due to the embryonic lethality of conventional Smad2 and Smad4 knockout mice, we previously used conventional Smad3 knockout (Smad3KO) mice to study the role of the TGF $\beta R /$ Smad signaling pathway in LC development. We found that the TGF $\beta R /$ Smad3 signaling pathway was inessential for LC development (16). Therefore, it raises the possibility that Smad2 or Smad4 may be involved in TGF $\beta 1$-mediated LC development. Human Langerin ${ }^{\mathrm{Cre}}\left(\mathrm{hLan}{ }^{\mathrm{Cre}}\right)$ and $\mathrm{Csf1} \mathrm{r}^{\mathrm{Cre}}$ reporter mice are reliable tools to study LCs in mice. hLan ${ }^{\text {Cre }}$ induces LC-exclusive gene deletion after birth, while Csf1 $\mathrm{r}^{\mathrm{Cre}}$ induces gene deletion in monocytes and macrophages in addition to LCs $(4,9)$. Here, we generated the mice with conditional deletions of either Smad2 or Smad4, or both Smad2 and Smad4 in LCs, mediated by either hLan ${ }^{\mathrm{Cre}}$ or Csf1r ${ }^{\mathrm{Cre}}$. We compared the frequency and function of LCs between knockout (KO) and wild type (WT) littermates. Similar to our previous observations in Smad3KO mice, the Smad2/4 signaling pathway was not required for LC maintenance, maturation, antigen uptake, or migration in vivo or in vitro in the steady state. We found that Smad2/4 signaling was involved in BM-derived LC replenishment after UV exposure. Therefore, our results further demonstrate that TGF $\beta 1$ regulates LC homeostasis through a Smad4-independent pathway during steady state and suggest that TGF $\beta 1$ regulates LC repopulation through a Smad4-dependent pathway during the inflammatory state.

\section{MATERIALS AND METHODS}

\section{Mice}

Csf1r ${ }^{\text {Cre }}$ mice (\#021024) and Smad4 $4^{\mathrm{fl} / \mathrm{fl}}$ mice $(\# 017462)$ were purchased from Jackson Laboratory. The Smad2 $2^{\mathrm{fl} / \mathrm{fl}}$ mice were kind gifts from Dr. Daniel Bernard at McGill University. Human Langerin ${ }^{\mathrm{Cre}}$ mice (9) were reported previously. All mice were backcrossed to C57BL/6J genetic background for at least six generations. The experiments were conducted in 7- to 12 -weekold mice (both sexes) unless otherwise indicated. The mice were bred and maintained in a pathogen-free facility at the Laboratory Animal Services Center of Henry Ford Health System. Handling of mice and experimental protocols were in accordance with the Institutional Animal Care and Use Committee of Henry Ford Health System.

\section{Epidermal Langerhans Cells Suspension Preparations}

Mouse skin was harvested and processed as previously described (17). Briefly, the separated epidermal sheet was first incubated in $0.25 \%$ Dispase (Gibco, Japan) for $1 \mathrm{~h}$ at $37^{\circ} \mathrm{C}$. Next, the epidermal sheet was briefly digested in RPMI (HyClone, Logan) containing 10\% FBS (ATLANTA biologicals, Flowery Branch) and $0.01 \%$ DNase I (Worthington, Lakewood). The sample was filtered through a $40 \mu \mathrm{m}$ mesh, and epidermal single cells were collected for flow cytometry.

\section{Lymph Node Suspension Preparations}

Lymph nodes (LNs) were harvested and incubated in PBS containing 3\% FBS, $1 \mathrm{mg} / \mathrm{mL}$ collagenase D (Roche Diagnostics, Germany), and $100 \mathrm{U} / \mathrm{mL}$ DNase I (Worthington, Lakewood) for $20 \mathrm{~min}$ at $37^{\circ} \mathrm{C}$. The sample was filtered through a $40 \mu \mathrm{m}$ mesh and LN single cells were collected for flow cytometry.

\section{LC Antigen Uptake in vitro}

Dextran-Fluorescein isothiocyanate isomer I (FITC) (Life Technologies, NY) was added into the epidermal suspension cells to a final concentration of $0.025 \%$. The epidermal cells were shaken and incubated for $45 \mathrm{~min}$ at either 4 or $37^{\circ} \mathrm{C}$. Following incubation, the epidermal cells were collected for flow cytometry analysis (18). 


\section{LC Culture in vitro}

Freshly-isolated epidermal cells were suspended in RPMI containing $10 \% \mathrm{FBS}, 5.5 \times 10^{-5}$ 2-Mercaptoethanol (Gibco, Grand Island), $0.02 \mathrm{M}$ HEPES buffer (Corning, Manassas), 1X MEM Non-essential Amino Acids (Gibco), $1 \mathrm{mM}$ Sodium Pyruvate (Gibco), $100 \mathrm{U} / \mathrm{mL}$ Penicillin, and $100 \mu \mathrm{g} / \mathrm{mL}$ Streptomycin, then were incubated for $72 \mathrm{~h}$ at $37^{\circ} \mathrm{C}$. Following incubation, the cells were collected for flow cytometry analysis.

\section{LC Migration and Phagocytosis in vivo}

Mouse ear $(10 \mu \mathrm{L})$ or belly $(200 \mu \mathrm{L})$ was painted with $5 \mathrm{mg} / \mathrm{mL}$ FITC (Sigma, St. Louis) in acetone/dibutyl phthalate (1:1). After $24 \mathrm{~h}$ of treatment, the skin-draining LNs were collected for flow cytometry analyses (18).

\section{Flow Cytometry}

Cells were resuspended and mixed with anti-CD16/32 (clone $2.4 \mathrm{G} 2$ ) for $10 \mathrm{~min}$ at $4^{\circ} \mathrm{C}$, then stained with the following antibodies: CD80 (16-10A1), CD86 (GL-1), Langerin (4C7), MHCII (M5/114.15.2), CD45 (30-F11), and EpCAM (G8.8). All antibodies used were from Invitrogen, eBioscience, Tonbo, BD Biosciences, or BioLegend. The cells were acquired or sorted using a Becton Dickinson FACSAria II flow cytometer (BD Biosciences, San Jose). Data were analyzed in live cells using the FlowJo software after gating out double cells (Tree Star, Ashland).

\section{RNA Extraction and qRT-PCR}

RNA was isolated using the GenElute ${ }^{\mathrm{TM}}$ Total RNA Purification Kit (Sigma). qRT-PCR was performed using the QuantStudio 7 Flex Real-Time PCR System. Quantitative gene expression data were normalized to the expression of GAPDH. Primers used are as below: GAPDH F5' - GGTGAAGGTCGGTGTGA ACG-3' R5' - TGTAGACCATGTAGTTGAGGTCA-3'; Smad2 F5' - CCCACTCCATTCCAGAAAAC-3' R5'- GAGCCTGTG TCCATACTTTG-3'; Smad4 F5' - CCAGGATGGTGGACTATG AAAT - $3^{\prime}$ R5' - GCAGCAAACACATCTCTCAAC - $3^{\prime}$.

\section{Immunofluorescence}

Mouse ear tissue was first floated on 20 mM EDTA (AMRESCO, Ohio) overnight at $4^{\circ} \mathrm{C}$. Then, the epidermal sheets were detached from the dermis and fixed in acetone for $15 \mathrm{~min}$ at $-20^{\circ} \mathrm{C}$ and placed on slides. The samples were blocked by $1 \%$ anti-CD16/32 (clone 2.4G2) in 3\% BSA and stained with $1 \%$ MHCII (M5/114.15.2). The slides were examined with an Olympus FSX100 microscope.

\section{LC Replenishment After Skin Inflammation}

The mice were anesthetized, and the shaved back was exposed to ultraviolet (UV) light for $15 \mathrm{~min}$ (wavelength: $254 \mathrm{~nm}$; voltage: $8 \mathrm{~W}$; source: $38 \mathrm{~cm}$ ) as previously described (18). The mice were sacrificed at day 5 or day 20 post-UV treatment for LC replenishment analysis.

\section{Statistical Analysis}

Statistical analysis was performed with Prism 7.0 (GraphPad, La Jolla, Calif) and SPSS (IBM, NY). Data were analyzed using the two-tailed Student's $t$-test when variances were equal; otherwise, the unpaired $t$-test with Welch correction was used. A $p$-value of $<0.05$ was considered to be statistically significant.

\section{RESULTS}

\section{Smad2 Is Not Required for LC Maintenance}

We previously reported that TGF $\beta \mathrm{R} / \mathrm{Smad} 3$ signaling pathway was inessential for LC development (16). To investigate the role of $\mathrm{Smad} 2$ in $\mathrm{LC}$ regulation, we crossed $\mathrm{Smad} 2 \mathrm{fl} / \mathrm{fl}$ mice with hLan ${ }^{C r e}$ mice (19) to generate mice with LC-exclusive Smad2 deletion after birth. Expression of Smad2 in epidermal LCs from hLan ${ }^{\mathrm{Cre}} \mathrm{Smad} 2^{\mathrm{fl} / \mathrm{fl}}$ mice (hSmad2KO) was dramatically reduced compared to WT littermates; this was confirmed by quantitative RT-PCR (qRT-PCR) (Figure 1A). LCs are the only epidermal $\mathrm{CD}_{4}{ }^{+} \mathrm{MHCII}^{+}$population in the epidermis at the steady state. As shown in Figure 1B, there was no significant alteration in the ratio or number of epidermal $\mathrm{LCs}\left(\mathrm{CD} 45^{+} \mathrm{MHCII}^{+}\right)$between WT and hSmad2KO mice; this was analyzed by flow cytometry. Therefore, the specific loss of Smad2 in the LCs had no significant effect on LC maintenance.

\section{Smad2 Deficiency Does Not Hamper LC Maturation}

During cell maturation, LCs have increased expression of MHCII and cell membrane costimulatory molecules, such as CD80 and CD86. Prior research has shown that LC-specific deletion of either TGF $\beta 1$, TGF $\beta$ R1, or TGF $\beta$ R2 results in spontaneous LC maturation and increased expression of CD80 and CD86 (19, 20). Given these research findings, we next examined whether Smad2 is necessary for maintaining LC immaturity. As shown in Figure 1C, the expression of MHCII, CD80, and CD86 in LCs were unaltered in hSmad2 $\mathrm{KO}$ mice compared to WT mice at steady state. This finding contrasts previous observation that LCs spontaneously matured in the mice with the deletion of TGF $\beta 1$ or its receptors $(19,20)$. Similar to WT mice, LCs from KO mice had increased expression of MHCII, CD80, and CD86 after $72 \mathrm{~h}$ of in vitro culture, and there was no significant difference in the frequency and MFI (median fluorescence intensity) of MHCII, CD80, or CD86 in LCs between KO and WT mice, respectively, after $72 \mathrm{~h}$ of in vitro culture (Figures 1C,D). These observations indicate that, like Smad3 (16), Smad2 is not essential to maintain LC immaturity during steady state or for LC maturation upon stimulation.

\section{Smad4 Is Not Required for LC Homeostasis or Maturation at Steady State} Phosphorylated Smad2 and Smad3 lead to the formation of a heterotrimeric complex with Smad4 that translocates to the nucleus to regulate TGF $\beta 1$ target genes $(12,13)$. To further investigate the role of the TGF $\beta 1 / \mathrm{Smad}$ pathway, especially for Smad4 in LC development, hLan ${ }^{\text {Cre }}$ mice were crossed with $\mathrm{Smad} 4{ }^{\mathrm{fl} / \mathrm{fl}}$ mice to generate LC-specific Smad4 KO mice (hSmad4KO). The deletion of Smad4 in epidermal LCs was confirmed by qRT-PCR (Figure 1E). As shown in Figure 1F, there was no significant difference in the epidermal LC ratio or the LC number between hSmad4KO and WT littermates. Therefore, Smad4 is not required for LC homeostasis. Next, we 
A

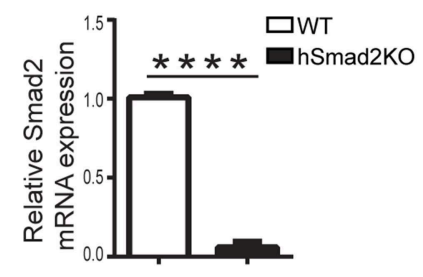

C
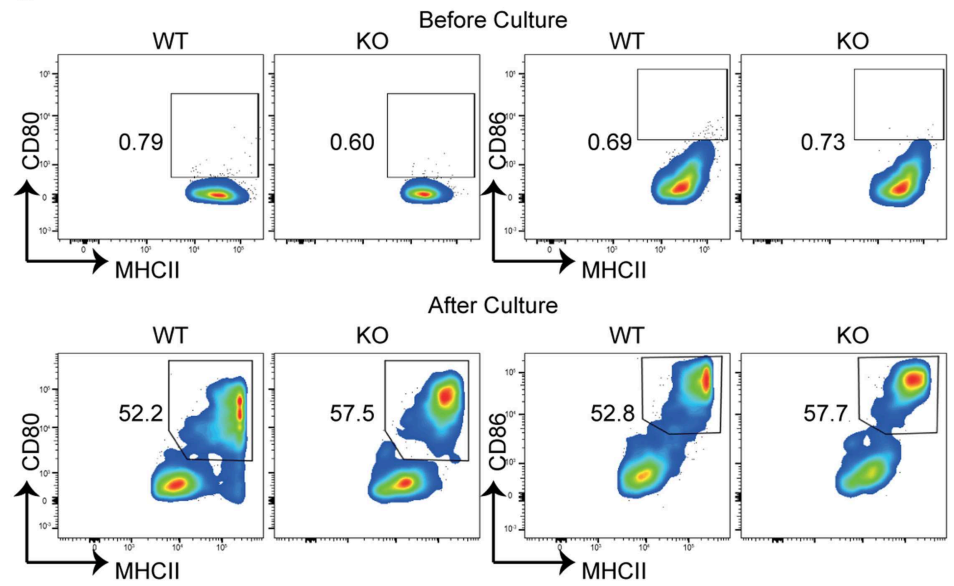

E

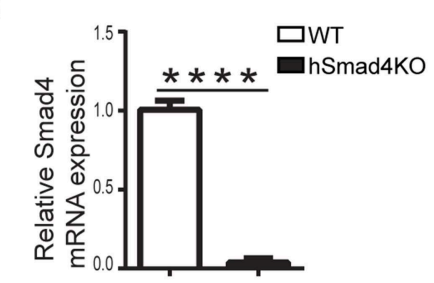

G

$\mathbf{F}$
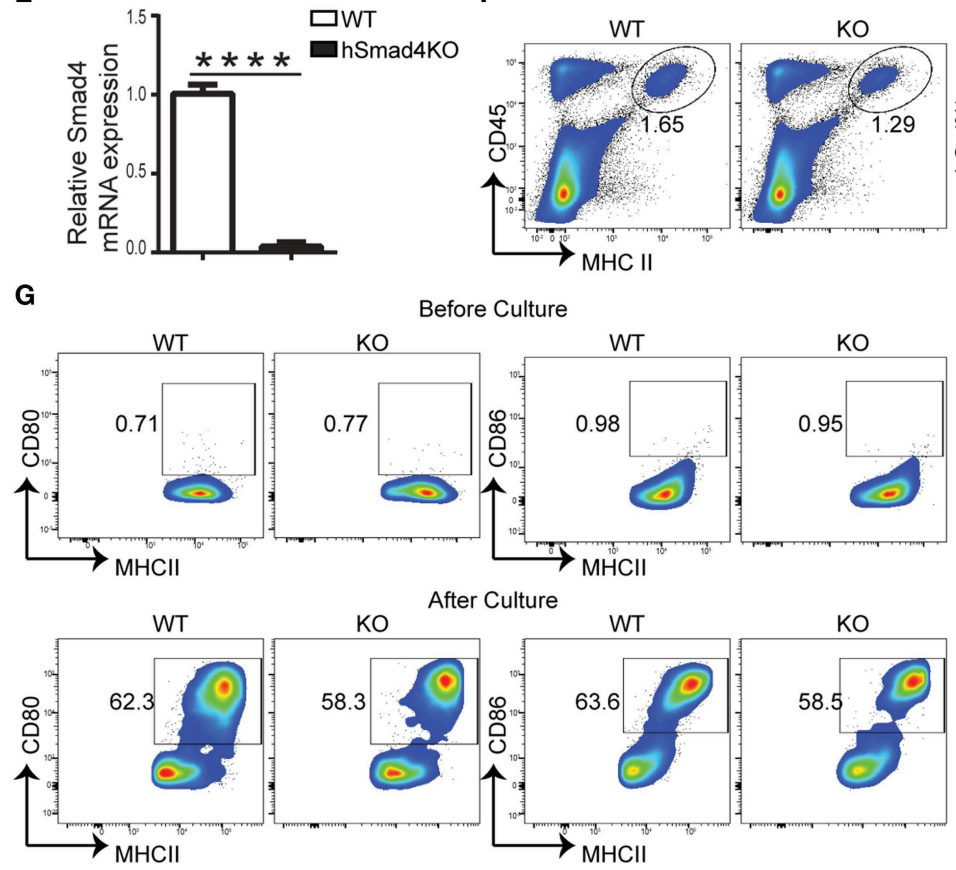
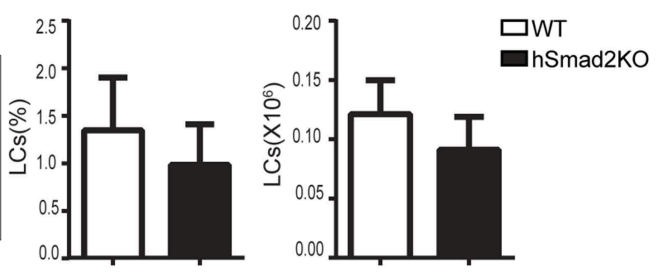

D
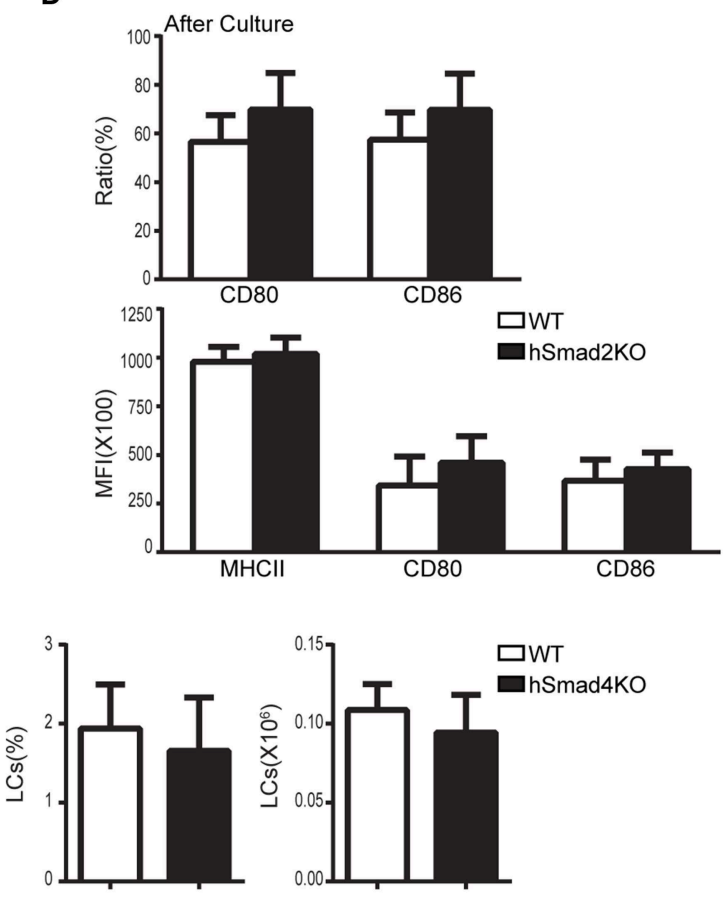

H
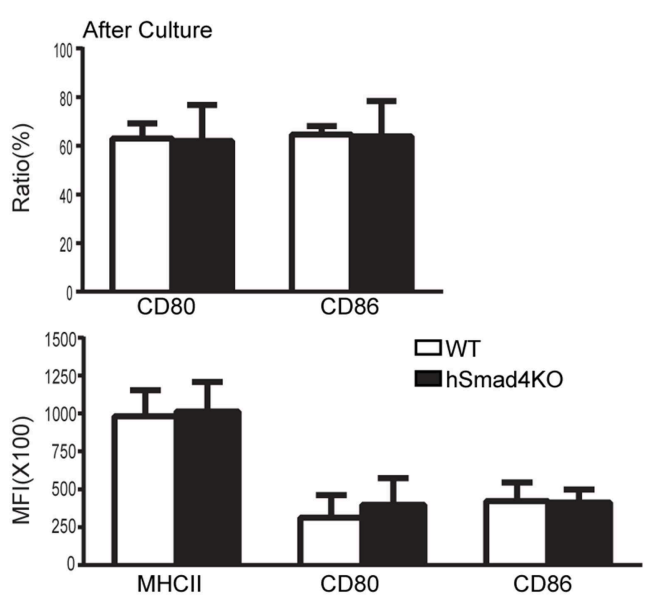

FIGURE 1 | Smad2 or Smad4 deficiency does not impact LC development or maturation. (A-D) In hSmad2KO and WT mice: (A) Expression of Smad2 in sorted LCs $\left(\mathrm{CD} 45^{+} \mathrm{MHCll}^{+}\right)$from $\mathrm{hSmad} 2 \mathrm{KO}$ and WT mice by qRT-PCR $\left(n=6,{ }^{* * \star *} P<0.0001\right)$. Frequencies and number of $\mathrm{LCs}\left(\mathrm{CD} 45^{+} \mathrm{MHCl}{ }^{+}\right)(\mathbf{B})$ and related maturation markers (C-top) from hSmad2KO mice and WT littermates at steady state $(n=8, P>0.2)$. Flow cytometric analyses of LCs (C-bottom), the frequency of MHCII, CD80 and CD86 and their expression of MFI (median fluorescence intensity) (D) after $72 \mathrm{~h}$ of in vitro culture were shown $(n=6-11, P>0.1)$. (E-H) In hSmad4KO and WT mice: (E) Expression of Smad4 in sorted LCs from hSmad4KO and WT mice by qRT-PCR $\left(n=6\right.$, $\left.{ }^{\star \star \star \star} P<0.0001\right)$. (F,G) Frequency, number (F) and maturation (G-top) of LCs from hSmad4KO mice and their WT littermates at steady state ( $n=10, P>0.3)$. Flow cytometric analyses of LCs after $72 \mathrm{~h}$ of in vitro culture (G-bottom). The frequency of MHCII, CD80 and CD86 and their expression (MFI) (H) after in vitro culture were shown ( $n=6-16, P>0.3)$. Data were shown as mean \pm SD. 
examined the expression of CD80 and CD86 in LCs at the steady state, and after $72 \mathrm{~h}$ of in vitro culture (Figures $\mathbf{1 G}, \mathbf{H}$ ), there was no significant difference between $\mathrm{hSmad} 4 \mathrm{KO}$ mice and WT littermates under both conditions. Therefore, our current data and the previous data from Smad3KO mice (16) further indicate that the Smad2/3/4 pathway is not essential for LC homeostasis or maturation.

\section{Smad2 Is Dispensable for LC Phagocytosis and Migration}

Epidermal LCs acquire and process antigens and migrate to skindraining LNs. Then, the LCs present the antigens to $\mathrm{T}$ cells and mediate antigen-specific immunity or immune tolerance (21-23). To evaluate the role of Smad2 in LC phagocytosis, we incubated freshly-isolated epidermal cells from hSmad2KO and WT mice with Dextran-Fluorescein isothiocyanate isomer I (FITC) for $45 \mathrm{~min}$ at 37 or $4^{\circ} \mathrm{C}$ (control). The LCs labeled with Dextran-FITC $\left(\mathrm{CD}_{4}{ }^{+} \mathrm{MHCII}^{+} \mathrm{FITC}^{+}\right)$were defined to have effective phagocytosis. The ratio and MFI of $\mathrm{FITC}^{+} \mathrm{LCs}$ were comparable between hSmad2KO and WT mice (Figures 2A,B). Next, we further investigated the role of Smad2 in LC antigen capture and migration in vivo by FITC painting (24). We analyzed the frequency of migratory $\mathrm{FITC}^{+}$LCs $\left(\mathrm{CD} 45^{+}\right.$ $\mathrm{MHCII}^{+}$Langerin ${ }^{+} \mathrm{EpCAM}^{+} \mathrm{FITC}^{+}$) in skin-draining LNs and observed no significant difference between hSmad2KO and WT mice (Figure 2C). Therefore, $\mathrm{Smad} 2$ deficiency has no significant impact on LC antigen uptake or migration. This finding is similar to the previous observation with Smad3 deficiency (16).

\section{Smad4 Is Not Essential for LC Phagocytosis and Migration}

We next examined the function of Smad4 in LC phagocytosis and migration. The freshly-isolated epidermal cells from $\mathrm{hSmad} 4 \mathrm{KO}$ and WT mice were incubated with Dextran-FITC, and we found that there was no significant difference in the frequency or MFI of FITC $^{+}$LCs between hSmad4KO mice and WT littermates (Figures 2D,E). Therefore, LC-specific Smad4 deletion had no significant effect on LC antigen-capture capabilities in vitro. We next examined the potential impact of Smad4 deficiency on LC antigen capture and migration in vivo. After painting the skin with FITC, draining LNs were harvested and analyzed by flow cytometry. There was no significant difference in the frequency of migrated $\mathrm{FITC}^{+}$LCs $\left(\mathrm{CD} 45^{+} \mathrm{MHCII}^{+}\right.$ Langerin $^{+}$EpCAM $^{+}$FITC $^{+}$) between hSmad4KO and WT mice (Figure 2F). Therefore, Smad4 is not essential for either LC phagocytosis or migration in both in vivo and in vitro models. These findings are consistent with the observations in hSmad2KO mice.

\section{Smad2/4 Pathway Is Not Required in LC Development and Function}

To exclude the possibility of redundancy among Smad2, Smad3, and Smad4, we attempted to generate Csf1r ${ }^{\mathrm{Cre}}$-induced conditional Smad2\&3 double KO, Smad3\&4 double KO, and triple $\mathrm{KO}$ mice; unfortunately, we were unsuccessful in making them so far. However, we were able to make Csf1r ${ }^{\text {Cre }} \operatorname{Smad} 2^{\mathrm{fl} / \mathrm{fl}}$
$\mathrm{Smad} 4{ }^{\mathrm{fl} / \mathrm{fl}}$ mice (cSmad2\&4KO) that had a simultaneous deletion of both Smad2 and Smad4. The conditional deletion of Smad2 and Smad4 on LCs was confirmed by qRT-PCR (Figure 3A). Immunohistochemical staining of the epidermal sheets showed no significant difference in the number of $\mathrm{MHCII}^{+} \mathrm{LCs}$ between $\mathrm{cSmad} 2 \& 4 \mathrm{KO}$ and WT mice (Figure $3 \mathrm{~B}$ ). In addition, FACS analyses further showed that there was no significant difference in the expression of MHCII, CD80, or CD86 in LCs between $\mathrm{cSmad} 2 \& 4 \mathrm{KO}$ mice and WT littermates before and after $72 \mathrm{~h}$ of culture (Figures 3C,D). Next, we evaluated LC antigen uptake and migration in vitro and in vivo, and observed no difference between them (Figure 3E,F). This was consistent with the results from individual hSmad2KO and hSmad4KO mice, further suggesting that the Smad2/4 pathway is not required in LC development at steady state. Given that Smad3 is not required for LCs (16), our data highly suggests that the Smad2/3/4 pathway is dispensable for LC maintenance, maturation, antigen uptake, and migration during the steady state.

\section{Smad2/4 Signaling Pathway Is Critical for "Long-Term" LC Repopulation During Inflammation}

Two waves of LC repopulation exist in the skin under inflammation conditions, including "short-term" LCs, which are derived from peripheral blood Gr- ${ }^{\text {hi }}$ monocytes and express low levels of Langerin, and "long-term" LCs, which are derived from BM and express high levels of Langerin (8). Previous studies established a reliable mouse model treated with UVC to study LC regeneration under inflammation conditions, and uncovered that inhibition of differentiation and DNA binding 2 (ID2) was involved in "long-term" LC repopulation but not in "shortterm" LC repopulation (8). To explore the role of the Smad2/4 signaling pathway in LC repopulation, we depleted epidermal LCs in cSmad2\&4KO and WT mice by UVC treatment (8). The conditional deletion of Smad2 and Smad4 on BM-derived macrophages was confirmed by qRT-PCR (Figure S1), suggesting a specific myeloid cell lineage deletion. In addition, lack of Smad2/4 significantly blocked lung macrophage development in the embryonic and after birth, indirectly indicating that Smad2/4 was functionally deleted in the myeloid lineage in cSmad $2 \& 4 \mathrm{KO}$ mice (unpublished data). We next compared the ratio of "shortterm" LCs $\left(\mathrm{CD}^{+} 5^{+} \mathrm{MHCII}^{+}\right.$Langerin $\left.^{-}\right)$and "long-term" LCs $\left(\mathrm{CD}^{+} 5^{+} \mathrm{MHCII}^{+}\right.$Langerin $\left.{ }^{+}\right)$between $\mathrm{KO}$ and WT littermates at day 5 and day 20 post-UVC treatment. As expected, UVC treament effectively depleted epidermal LCs and only about $10 \%$ epidermal LCs left at day 3 in WT mice post-treatment (Figure S2). As shown in Figure 4A, there was no significant difference in the frequency of replenished epidermal LCs between cSmad2\&4KO mice and WT controls at day 5. However, more than $95 \%$ of the LCs in cSmad $2 \& 4 \mathrm{KO}$ mice were Langerin ${ }^{-}$ "short-term" LCs and a few were Langerin " "long-term" LCs, while about $50 \%$ of the LCs in WT mice were Langerin " "shortterm" LCs and about 50\% were Langerin " "long-term" LCs (Figure 4B). Whereas, a significant reduction in the frequency of replenished epidermal LCs in $\mathrm{cSmad} 2 \& 4 \mathrm{KO}$ mice compared 


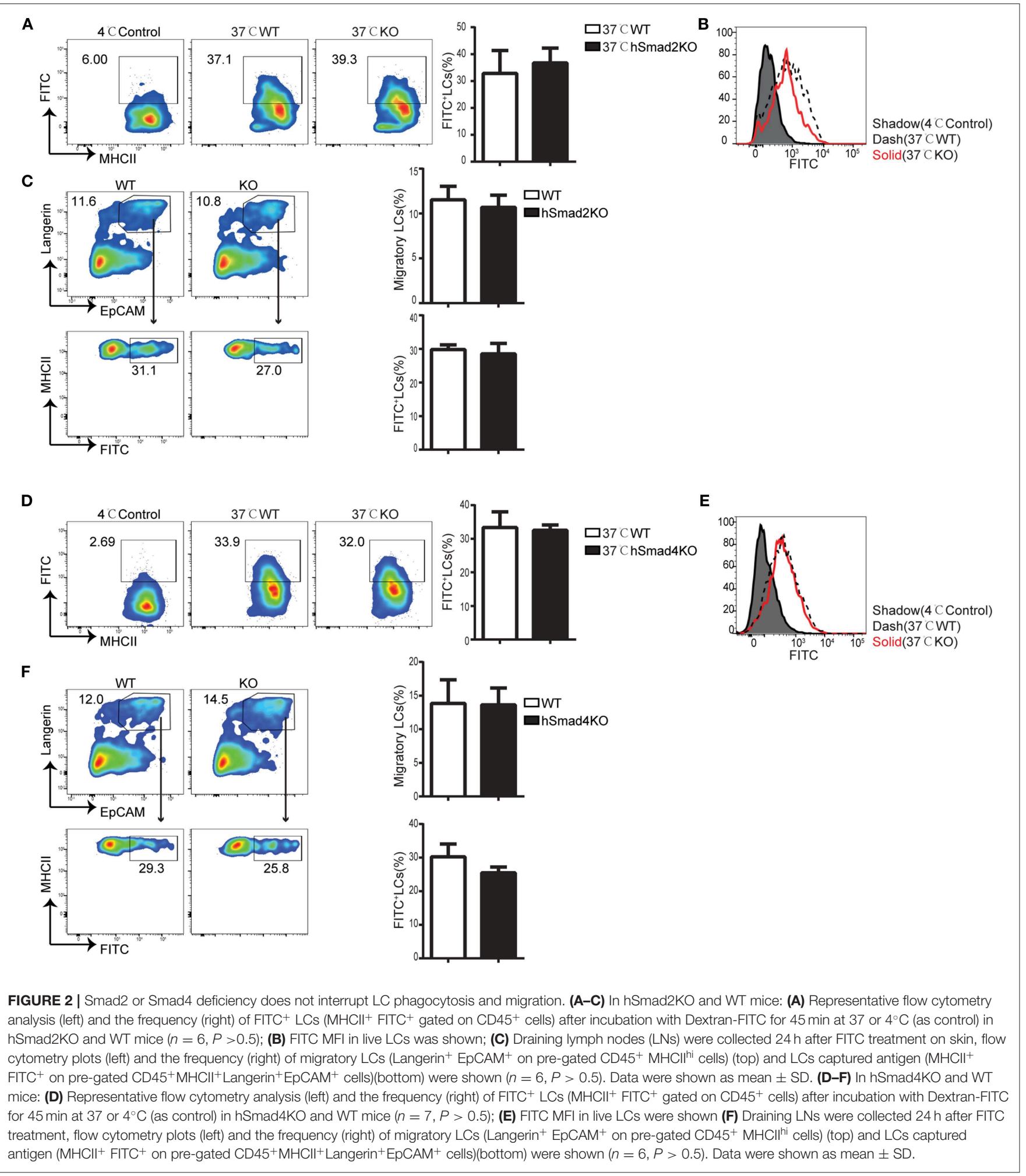

to WT controls at day 20 was observed (Figure 4C), most of the LCs from cSmad2\&4KO mice were Langerin- "shortterm" LCs $(>80 \%)$. In contrast, very few LCs in WT mice were Langerin" "short-term" LCs $(<10 \%)$ (Figure 4D). We also analyzed the LCs in skin drain LNs at day 5 and day
20 post-UVC treatment, as showed in Figure S3, there was no significant difference on LC frequency and number between $\mathrm{KO}$ and WT mice. Long-term LCs are discriminated from short-term LCs by more abundant expression of EpCAM and CD24 on long-term LCs (8). As shown in Figure S4, the expression of 

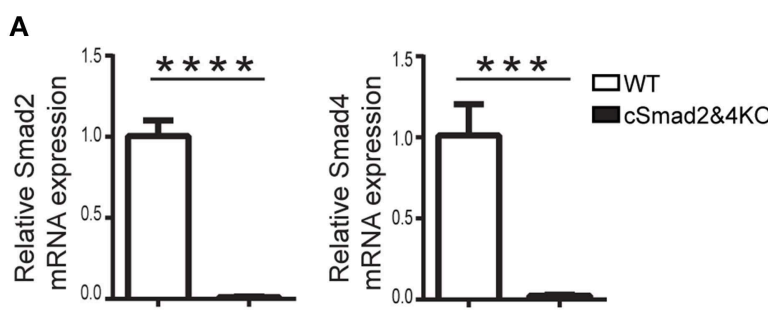

C
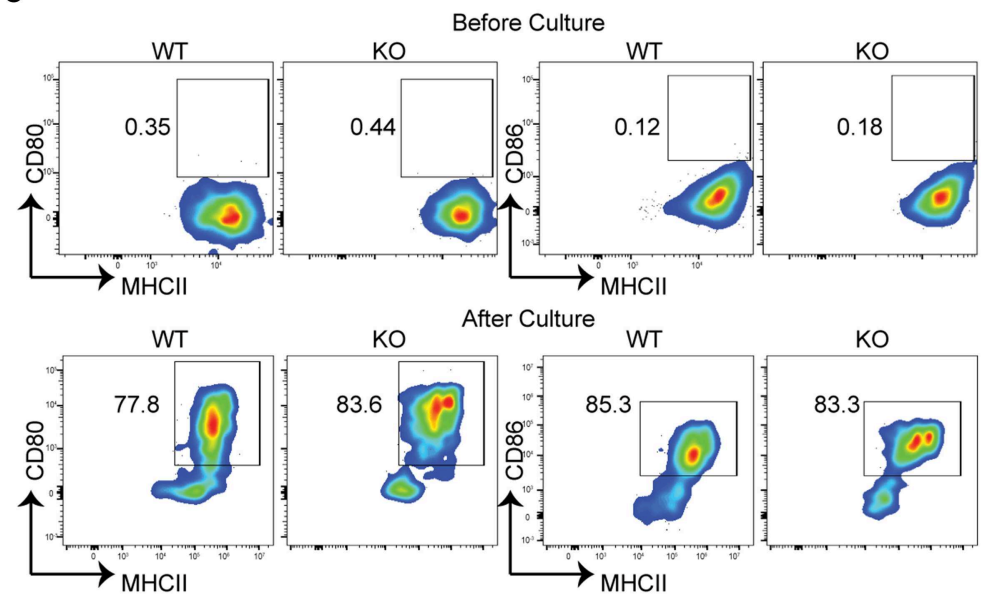

$\mathbf{E}$

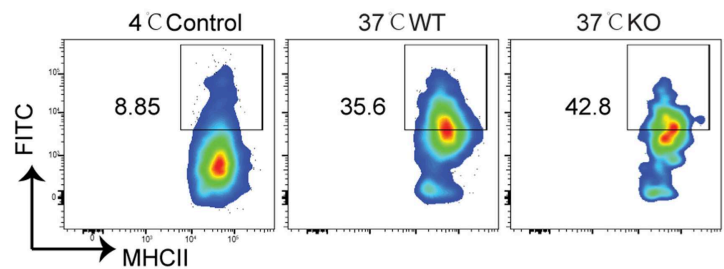

$\mathbf{F}$
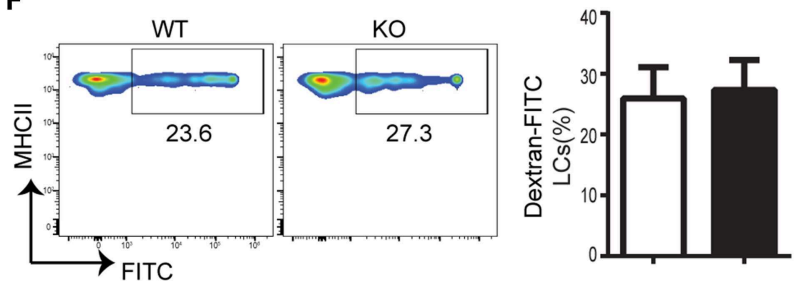

$\square 37$ CWT

$\square 37$ C cSmad2\&4KO

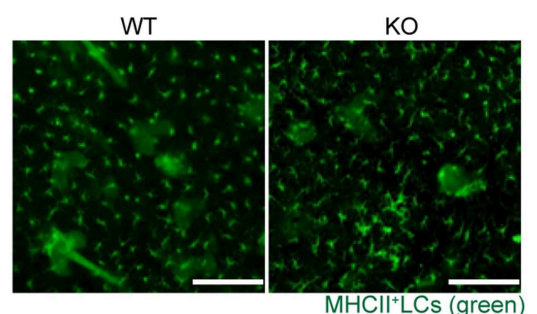

D

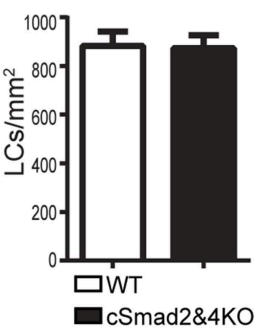

After Culture
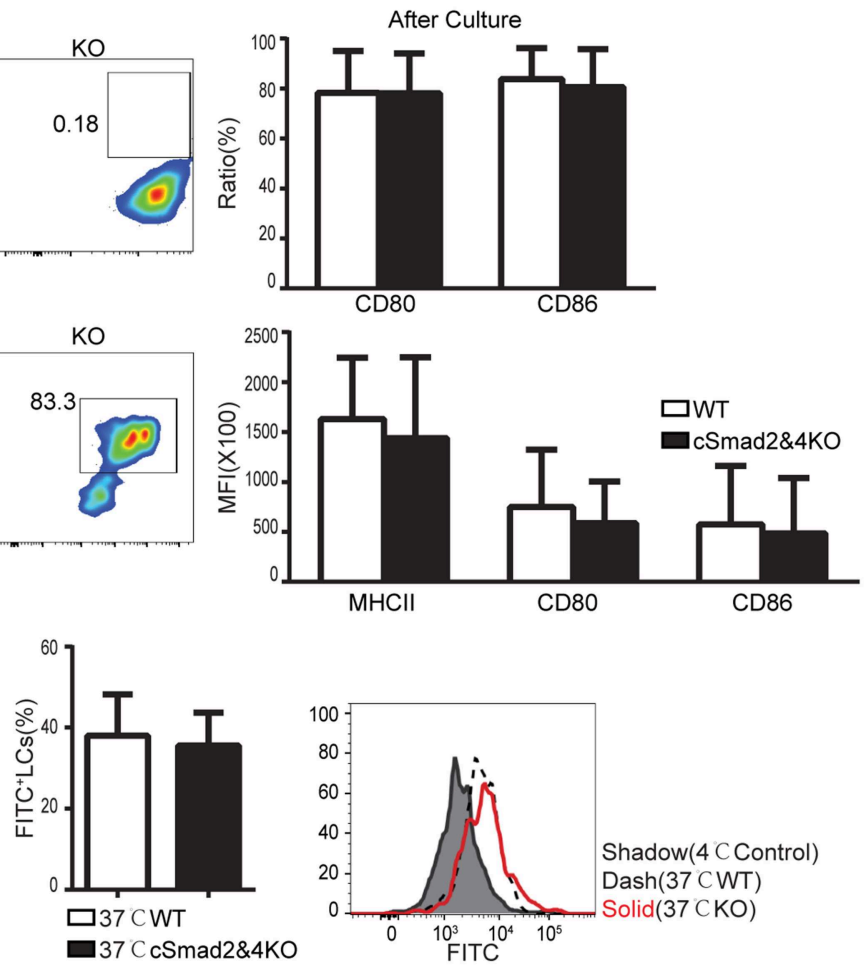

FIGURE 3 | Smad2/4 signaling is not essential for LC homeostasis, maturation, phagocytosis, and migration. In cSmad2\&4KO and WT mice, (A) Expression of Smad2 and Smad4 in sorted LCs by qRT-PCR $\left(n=6,{ }^{\star \star \star} P=0.0009\right.$, $\left.{ }^{\star \star \star \star} P<0.0001\right)$. (B) Epidermal sheets at 5 weeks old were stained with anti-MHCll (green). Scale bar $=100 \mu \mathrm{m}$, original magnification X10 $(n=6, P=0.8484)$. (C,D) Flow cytometry analyses of LCs before and after $72 \mathrm{~h}$ of in vitro culture, the frequency of MHCII, CD80 and CD86 (C) and their expression (D) $(n=6-18, P>0.5)$ were shown. (E) The frequency of FITC ${ }^{+}$LCs after incubation with Dextran-FITC for 45 min at 37 or $4^{\circ} \mathrm{C}$ (as control) and FITC MFI in live LCs were shown $(n=6, P=0.7591)$. (F) The frequency of FITC ${ }^{+}$LCs in pre-gated migratory $L C s$ in $L N s(n=7, P=$ 0.7301). Data were shown as mean $\pm S D$

$\mathrm{EpCAM}^{+}$and $\mathrm{CD}_{2} 4^{+}$on "long-term" Langerin ${ }^{+} \mathrm{LCs}$ were dramatically reduced in Csf1 ${ }^{\mathrm{Cre}} \operatorname{Smad} 2^{\mathrm{fl} / \mathrm{fl}}(\mathrm{cSmad} 2 \mathrm{KO})$ mice at day 5 post-UVC treatment, while the expression of $\mathrm{EpCAM}^{+}$and $\mathrm{CD}_{24}{ }^{+}$on short-term Langerin ${ }^{-}$LCs were comparable between cSmad2KO and WT mice. Overall, the Smad2/4 signaling pathway is not required for the first wave of "short-term" LC repopulation derived from peripheral monocytes, but is required for the second wave of "long-term" repopulation from BMderived progenitors.

\section{DISCUSSION}

TGF $\beta 1$ plays a central role in immune system development through various signaling pathways, which includes the 

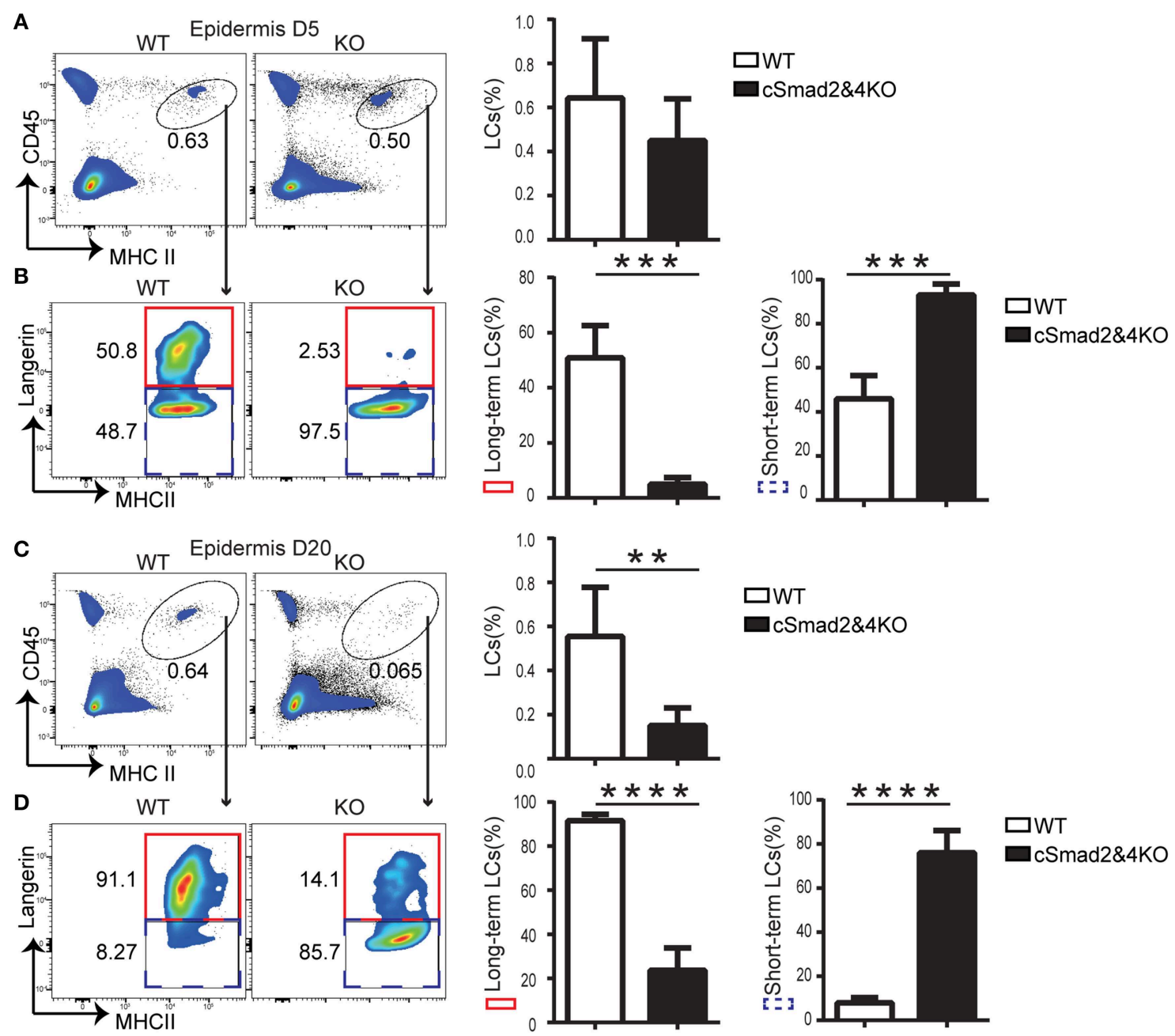

FIGURE 4 | Smad2/4 signaling is required for "long-term" LC repopulation. (A,B) Smad2/4 signaling pathway is not required for "short-term" LC repopulation. (A) The frequency of LCs at day 5 (D5) after UV treatment ( $n=9, P=0.31$ ); (B) The frequency of peripheral monocytes-derived "short-term" $L C s$ (blue dashed line) and bone marrow-derived "long-term" LCs (red solid line) on pre-gated live LCs $\left(n=9\right.$, $\left.{ }^{\star \star \star} P<0.0002\right)$. (C,D) Smad2/4 signaling pathway is required for "long-term" LC repopulation. (C) The frequency of LCs at day 20 (D20) after UV treatment $\left(n=12\right.$, $\left.{ }^{\star *} P=0.0019\right)$; (D) The frequency of peripheral monocytes-derived "short-term" LCs (blue dashed line) and bone marrow-derived "long-term" LCs (red solid line) on pre-gated LCs $\left(n=12,{ }^{* \star \star *} P<0.0001\right)$. Data were shown as mean \pm SD.

canonical Smad2/3/4 pathway and non-canonical Smadindependent pathways (25). TGF $\beta 1$ is a crucial regulator of LC homeostasis and function after birth. Mice that lack TGF $\beta 1$, TGF $\beta R 1$, or TGF $\beta R 2$ exhibit a profound reduction in epidermal LCs $(9,10,20)$. However, the specific underlying roles of TGF $\beta 1$ signaling in LC development and function remain unclear. Our previous research has shown that the TGF $\beta / \mathrm{Smad} 3$ signaling pathway does not impact LC function or homeostasis (16). To further explore the role of TGF $\beta /$ Smad signaling pathways in LC maintenance and function, in this study, we first generated mice with LC-specific conditional deletion of Smad2 or Smad4, and found that LC-specific deletion of Smad2, Smad4, or both Smad2 and Smad4 had no significant effect on epidermal LC homeostasis during steady state. In addition, our preliminary data also suggests that the $\operatorname{Smad} 2 / 4$ pathway is not required for LC embryonic development as well as newborn pups either (unpublished data). Previous studies have shown that
TGF $\beta 1$ regulates LC maturation and migration (19, 20). The inhibition of TGF $\beta 1$ and its signaling pathways can trigger LCs to spontaneously mature and migrate to skin-draining LNs $(19,20)$. No significant difference was observed in LC maturation and migration between $\mathrm{KO}$ mice and $\mathrm{WT}$ controls during in vivo assays. Furthermore, there was no significant difference in the expression of MHCII, CD80, or CD86 between KO and WT mice upon in vitro stimulation. Taken together with our previous results studying Smad3 (16), our current data suggests that canonical TGF $\beta / S m a d 2 / 3 / 4$ signaling pathways are not required for maintaining epidermal LC homeostasis and immaturity at the steady state.

TGF $\beta$ receptors are known to also mediate a variety of non-Smad signaling pathways, which include mitogen-activated protein kinase (MAPK), extracellular signal-regulated kinases (Erks), c-Jun amino terminal kinase (JNK), p38 МАРК, ІкВ kinase (IKK), phosphatidylinositol-3kinase (PI3K), Akt, and 
Rho family GTPases (26). Recent research has shown that the PI3K/Akt/mTOR pathway plays an important role in DC development and function. The regulatory protein $\mathrm{mTORC} 1 \mathrm{can}$ stimulate PI3K, which leads to downstream activation of Akt and PI3K. Prior research has shown that the conditional deletion of Raptor, a component of the mTORC1 complex, leads to the progressive loss of epidermal LCs (27). However, the loss of mTORC1 activity did not affect the initial epidermal seeding with LCs in this study (27), which differs from the phenotype of TGF $\beta 1$ or its receptor loss in LCs $(19,20)$. Young mice with Raptor deficiency have increased LC migration out of the skin, resulting in increased migratory LC frequency inside skindraining LNs, which is similar to the phenotype in TGF $\beta 1$ or its receptor loss in LCs $(19,20)$. Therefore, the loss of epidermal LCs in mTORC1KO mice is due to increased migration out of the skin, which only partially reflects the phenotype of TGF $\beta 1$ or its receptor loss in LCs. The role of the TGF $\beta / \mathrm{PI} 3 \mathrm{~K} / \mathrm{Akt}$ pathway in LC homeostasis still needs to be clarified in future research. Activated TGF $\beta$ receptors are also known to interact with TRAF6 and TGF $\beta$-activated kinase 1 (TAK1). TAK1 activates several downstream kinases, such as JNK, p38 MAPK, and IKK. Interestingly, TAK1 is an essential regulator of DC homeostasis and function (28). Therefore, it would be very interesting to further investigate the role of TGF $\beta /$ TAK1/JNK/MAPK pathways in LC homeostasis and function in the future.

Like macrophages, under stress or inflammatory conditions, LCs may be generated from monocytes and BM-derived progenitors (29). There are two waves of LCs that repopulate the epidermis following UV-induced inflammation (8). The first wave involves Langerin " "short-term" LCs derived from Gr-1 ${ }^{\text {hi }}$ monocytes and is detected 5 days post-irradiation, which persists for up to 3 weeks; the second wave involves Langerin ${ }^{+}$"longterm" LCs derived from undefined precursors of BM, which later become the overwhelming majority LC population (8). TGF $\beta 1$ has been found to regulate BM-derived LC repopulation via interaction with the inhibitor of differentiation and DNA binding 2 (ID2) (8). In addition, TGF $\beta 1$ has been found to regulate $\mathrm{BM}$-derived $\mathrm{LC}$ repopulation via interaction with PU.1 in a RUNX-3-depedent pathway (30). Previous studies indicate that hLangerin ${ }^{\mathrm{Cre}}$ reporter mice only mapped welldeveloped epidermal LCs and migrated LCs in skin-draining LN exclusively, without influencing other cell lineages including Langerin ${ }^{+} \mathrm{CD}^{+}$DCs $(9,19)$. Thus, hLangerin ${ }^{\text {Cre }}$ could not mediate gene deletion in the Langerin ${ }^{-}$short-term LCs. Csf1r ${ }^{C r e}$ has been used to induce the gene deletion in the LCs as well as in the myeloid lineage cells, including monocytes and BM-derived macrophages (4). Thus, to investigate Smad pathways in LC repopulation under inflammatory conditions, we generated the myeloid lineage Smad2\&4 KO mice mediated by Csf1r ${ }^{\text {Cre }}$ rather than by hLangerin ${ }^{\mathrm{Cre}}$. In this study, we analyzed the frequency of repopulated LCs at day 5 and day 20 post-UV treatment using flow cytometry. We found that the conditional deletion of Smad2 and Smad4 together dramatically blocked the differentiation of $\mathrm{MHCII}^{+}$Langerin ${ }^{+}$"long-term" LCs during the inflammatory state, but had no significant effect on monocyte-derived $\mathrm{MHCII}^{+}$ Langerin " "short-term" LCs. Since Smad2 must activate Smad4 to regulate the Smad downstream pathway, as expected, the deletion of Smad2 in LCs also displayed the interruption of long-term LC repopulation and the lower expression of EpCAM and CD24 on the long-term LCs, while short-term LCs were unaffected. Ideally, immunohistochemical analysis of LCs in the epidermal sheets would be analyzed alongside flow cytometry, but the limited availability of harvestable skin makes parallel analysis technically difficult. However, given the severe defect on the number of LC repopulation in $\mathrm{CSmad} 2 \& 4 \mathrm{KO}$ mice by FACS analysis, our data strongly supports our conclusion that Smad4 pathway is required for long-term LC repopulation.

A recent study, using an allogeneic hematopoietic stem cell transplantation model where allo-reactive $\mathrm{T}$ cells directly target LCs to ask if and how the LC network is ultimately restored, reports that donor monocytes are the precursors of the long-term LCs that are detected 3 weeks after transferring (31). One possible explanation to the discrepancy is that that transient exposure to UV irradiation compared to the prolonged inflammation caused by allo-reactive $\mathrm{T}$ cells may trigger different mechanisms of $\mathrm{LC}$ repopulation in the skin. $\mathrm{Ly}_{6 \mathrm{C}}+$ monocytes are shortlived non-cycling cells, which can quickly repopulate to skin and differentiate into $\mathrm{MHCII}^{+}$Langerin ${ }^{-}$"short-term" LCs in UVinduced acute skin damage model, which are detected 5 days post-irradiation and only persist for up to 3 weeks. By contrast, in the stem cell transplant model, the allogeneic $\mathrm{T}$ cells are recruited to the epidermis over a period of weeks, resulting in prolonged immune pathology and inflammation. Under this condition, the monocytes can become long-term LC, contributing to the emerging LC network (31). It is possible that the precursors of long-term LCs in the transient exposure to UV irradiation might be $\mathrm{Ly} 6 \mathrm{C}^{-}$monocytes derived from BM, while short-term LCs, which needs to be further investigated in the future. In addition, the question that whether the Ly6 $\mathrm{C}^{+}$monocyte-derived "shortterm" LCs is an intermediate form of "long-term"LCs also needs further investigation under acute inflammation.

Both transcription factors PU.1 and ID2 play key roles in LC replenishment during inflammation. TGF $\beta 1$ is found to regulate BM-derived LC repopulation via interaction with the ID2 (8) or via interaction with PU.1 in a RUNX 3-dependent pathway (30). Thus, it is very likely that a Smad2/4-dependent pathway regulates PU.1 or ID2 activity during LC repopulation, which is currently under our investigation.

In conclusion, our data indicate that TGF $\beta 1$ regulates LC homeostasis and repopulation in both Smad4-dependent and Smad4-independent pathways. The Smad4 pathway is not required for LC homeostasis, maturation, antigen uptake, or migration during steady-state. In contrast, the Smad4 pathway is required for BM-derived "long-term" LC activity during inflammation. Based on the critical roles of LCs in skin immunity and diseases, our findings may help to develop novel therapeutics that target the TGF $\beta 1 / \mathrm{Smad} 4$ pathway in LC-related skin diseases.

\section{DATA AVAILABILITY STATEMENT}

All datasets generated for this study are included in the article/Supplementary Material. 


\section{ETHICS STATEMENT}

The animal study was reviewed and approved by Institutional Animal Care and Use Committee of Henry Ford Health System. Written informed consent was obtained from the owners for the participation of their animals in this study.

\section{AUTHOR CONTRIBUTIONS}

LH and G-HL performed most of the experiments. Q-SM, ZY, LZ, and DK conceived and designed the experiments. LH, QY, NP, and YX analyzed the data. SC, XW, and IU-I assisted with animal maintenance and genotyped mutant mice. LH, Q-SM, ZY, and LZ drafted the manuscript.

\section{REFERENCES}

1. Doebel T, Voisin B, Nagao K. Langerhans cells - The macrophage in dendritic cell clothing. Trends Immunol. (2017) 38:817-28. doi: 10.1016/j.it.2017.06.008

2. Valladeau J, Ravel O, Dezutter-Dambuyant C, Moore K, Kleijmeer M, Liu $\mathrm{Y}$, et al. Langerin, a novel C-type lectin specific to Langerhans cells, is an endocytic receptor that induces the formation of Birbeck granules. Immunity. (2000) 12:71-81. doi: 10.1016/S1074-7613(00)80160-0

3. Romani N, Clausen BE, Stoitzner P. Langerhans cells and more: langerinexpressing dendritic cell subsets in the skin. Immunol Rev. (2010) 234:120-41. doi: 10.1111/j.0105-2896.2009.00886.x

4. Yao Y, Martin C, Yin C, Guo C, Dong Z, Zhou L, et al. Micro RNAs are required for Langerhans cell, skin- and lung-resident macrophage ontogeny. $J$ Allergy Clin Immunol. (2018) 142:976-8.e2. doi: 10.1016/j.jaci.2018.04.024

5. Ginhoux F, Merad M. Ontogeny and homeostasis of Langerhans cells. Immunol Cell Biol. (2010) 88:387-92. doi: 10.1038/icb.2010.38

6. Hemmi H, Yoshino M, Yamazaki H, Naito M, Iyoda T, Omatsu Y, et al. Skin antigens in the steady state are trafficked to regional lymph nodes by transforming growth factor-betal-dependent cells. Int Immunol. (2001) 13:695-704. doi: 10.1093/intimm/13.5.695

7. Kaplan DH. Ontogeny and function of murine epidermal Langerhans cells. Nat Immunol. (2017) 18:1068-75. doi: 10.1038/ni.3815

8. Sere K, Baek JH, Ober-Blobaum J, Muller-Newen G, Tacke F, Yokota $\mathrm{Y}$, et al. Two distinct types of Langerhans cells populate the skin during steady state and inflammation. Immunity. (2012) 37:905-16. doi: 10.1016/j.immuni.2012.07.019

9. Kaplan DH, Li MO, Jenison MC, Shlomchik WD, Flavell RA, Shlomchik MJ. Autocrine/paracrine TGFbetal is required for the development of epidermal Langerhans cells. J Exp Med. (2007) 204:2545-52. doi: 10.1084/jem.20071401

10. Borkowski TA, Letterio JJ, Farr AG, Udey MC. A role for endogenous transforming growth factor beta 1 in Langerhans cell biology: the skin of transforming growth factor beta 1 null mice is devoid of epidermal Langerhans cells. J Exp Med. (1996) 184:2417-22. doi: 10.1084/jem.184.6.2417

11. Zahner SP, Kel JM, Martina CA, Brouwers-Haspels I, van Roon MA, Clausen BE. Conditional deletion of TGF-betaR1 using Langerin-Cre mice results in Langerhans cell deficiency and reduced contact hypersensitivity. J Immunol. (2011) 187:5069-76. doi: 10.4049/jimmunol.1101880

12. Tsukazaki T, Chiang TA, Davison AF, Attisano L, Wrana JL. SARA, a FYVE domain protein that recruits $S$ mad2 to the TGFbeta receptor. Cell. (1998) 95:779-91. doi: 10.1016/S0092-8674(00)81701-8

13. Derynck R, Zhang YE. Smad-dependent and Smad-independent pathways in TGF-beta family signalling. Nature. (2003) 425:577-84. doi: 10.1038 /nature02006

14. Zhang X, Gu J, Yu FS, Zhou L, Mi QS. TGF-beta1-induced transcription factor networks in Langerhans cell development and maintenance. Allergy. (2016) 71:758-64. doi: 10.1111/all.12871

\section{FUNDING}

This study was supported by grants from the Henry Ford Immunology Program (T71016) and the National Natural Science Foundation of China (81630083).

\section{ACKNOWLEDGMENTS}

We thank Dr. Daniel Bernard for providing Smad2 $2^{\mathrm{fl} / \mathrm{fl}}$ mice. We thank all laboratory members for their help and encouragement.

\section{SUPPLEMENTARY MATERIAL}

The Supplementary Material for this article can be found online at: https://www.frontiersin.org/articles/10.3389/fimmu. 2020.00912/full\#supplementary-material

15. Moustakas A, Heldin CH. Non-Smad TGF-beta signals. J Cell Sci. (2005) 118:3573-84. doi: 10.1242/jcs.02554

16. Xu YP, Shi Y, Cui ZZ, Jiang HH, Li L, Wang XF, et al. TGFbeta/Smad3 signal pathway is not required for epidermal Langerhans cell development. J Invest Dermatol. (2012) 132:2106-9. doi: 10.1038/jid.2012.71

17. Zhang $\mathrm{X}$, Yao $\mathrm{Y}$, Wei WZ, Yang ZQ, Gu J, Zhou L. Impaired epidermal Langerhans cell maturation in TGFbeta-inducible early gene 1 (TIEG1) knockout mice. Oncotarget. (2017) 8:112875-82. doi: 10.18632/oncotarget.22843

18. Zhang X, Liu Q, Wang J, Li G, Weiland M, Yu FS, et al. TIM-4 is differentially expressed in the distinct subsets of dendritic cells in skin and skin-draining lymph nodes and controls skin Langerhans cell homeostasis. Oncotarget. (2016) 7:37498-512. doi: 10.18632/oncotarget.9546

19. Bobr A, Igyarto BZ, Haley KM, Li MO, Flavell RA, Kaplan DH. Autocrine/paracrine TGF-betal inhibits Langerhans cell migration. Proc Natl Acad Sci USA. (2012) 109:10492-7. doi: 10.1073/pnas.1119178109

20. Kel JM, Girard-Madoux MJ, Reizis B, Clausen BE. TGF-beta is required to maintain the pool of immature Langerhans cells in the epidermis. J Immunol. (2010) 185:3248-55. doi: 10.4049/jimmunol. 1000981

21. Kaplan DH. In vivo function of Langerhans cells and dermal dendritic cells. Trends Immunol. (2010) 31:446-51. doi: 10.1016/j.it.2010. 08.006

22. Mi QS, Weiland M, Qi RQ, Gao XH, Poisson LM, Zhou L. Identification of mouse serum miRNA endogenous references by global gene expression profiles. PLoS ONE. (2012) 7:e31278. doi: 10.1371/journal.pone. 0031278

23. Mi QS, Xu YP, Wang H, Qi RQ, Dong Z, Zhou L. Deletion of microRNA miR-223 increases Langerhans cell cross-presentation. Int J Biochem Cell Biol. (2013) 45:395-400. doi: 10.1016/j.biocel.2012.11.004

24. Wang Z, Wang W, Chai Q, Zhu M. Langerhans cells control lymphatic vessel function during inflammation via LIGHT-LTbetaR signaling. J Immunol. (2019) 202:2999-3007. doi: 10.4049/jimmunol.1801578

25. David CJ, Massague J. Contextual determinants of TGFbeta action in development, immunity and cancer. Nat Rev Mol Cell Biol. (2018) 19:419-35. doi: 10.1038/s41580-018-0007-0

26. Zhang YE. Non-Smad signaling pathways of the TGF-beta family. Cold Spring Harbor Perspect Biol. (2017) 9:a022129. doi: 10.1101/cshperspect.a022129

27. Kellersch B, Brocker T. Langerhans cell homeostasis in mice is dependent on mTORC1 but not mTORC2 function. Blood. (2013) 121:298-307. doi: 10.1182/blood-2012-06-439786

28. Wang Y, Huang G, Vogel P, Neale G, Reizis B, Chi H. Transforming growth factor beta-activated kinase 1 (TAK1)-dependent checkpoint in the survival of dendritic cells promotes immune homeostasis and function. Proc Natl Acad Sci USA. (2012) 109:E343-52. doi: 10.1073/pnas.11156 35109 
29. Guilliams M, Scott CL. Does niche competition determine the origin of tissue-resident macrophages? Nat Rev Immunol. (2017) 17:451-60. doi: $10.1038 /$ nri.2017.42

30. Chopin M, Seillet C, Chevrier S, Wu L, Wang H, Morse HC III, et al. Langerhans cells are generated by two distinct PU.1dependent transcriptional networks. J Exp Med. (2013) 210:2967-80. doi: 10.1084/jem.20130930

31. Ferrer IR, West HC, Henderson S, Ushakov DS, Santos ESP, Strid J, et al. A wave of monocytes is recruited to replenish the long-term Langerhans cell network after immune injury. Sci Immunol. (2019) 4:eaax8704. doi: 10.1126/sciimmunol.aax8704
Conflict of Interest: The authors declare that the research was conducted in the absence of any commercial or financial relationships that could be construed as a potential conflict of interest.

Copyright (c) 2020 Huang, Li, Yu, Xu, Cvetkovski, Wang, Parajuli, Udo-Inyang, Kaplan, Zhou, Yao and Mi. This is an open-access article distributed under the terms of the Creative Commons Attribution License (CC BY). The use, distribution or reproduction in other forums is permitted, provided the original author(s) and the copyright owner(s) are credited and that the original publication in this journal is cited, in accordance with accepted academic practice. No use, distribution or reproduction is permitted which does not comply with these terms. 